\title{
Temporomandibular joint dislocation in a post-stroke patient with dysphagia caused by gastroesophageal reflux-related vomiting
}

\author{
Takahiro Kaneko, Miki Endo, Ayako Uematsu, Satoshi Nakamura, \\ Norio Horie, and Tetsuo Shimoyama \\ Department of Oral and Maxillofacial Surgery, Saitama Medical Center, Saitama Medical University, \\ Kawagoe, Japan
}

(Received April 7, 2015; Accepted September 27, 2015)

\begin{abstract}
Temporomandibular joint dislocation (TMJ) is sometimes found in post-stroke patients, and most cases occurs involuntarily. This report describes a rare case of repeated, voluntary, TMJ in a 73-yearold woman with dysphagia and dysarthria. The cause of dislocation was suspected to be voluntary excessive mouth opening associated with gastroesophageal reflux-related vomiting and the desire to eject oral deposits resulting from hypoglossal and facial nerve paralyses. After an oral hygiene intervention, the frequency of TMJ dislocation decreased and finally disappeared. Thorough oral hygiene seemed to contribute to protection against TMJ dislocation.
\end{abstract}

(J Oral Sci 58, 133-136, 2016)

Keywords: temporomandibular joint dislocation; stroke; dysphagia; oral hygiene.

\section{Introduction}

Temporomandibular joint (TMJ) dislocation involves displacement of the mandibular condyle from the mandibular fossa of the temporal bone. Common direct trigger factors for TMJ dislocation are yawning and treatments that require wide opening of the mouth, such as

Correspondence to Dr. Takahiro Kaneko, Department of Oral and Maxillofacial Surgery, Saitama Medical Center, Saitama Medical University, 1981 Kamoda, Kawagoe, Saitama 350-8550, Japan Fax: +81-49-228-3687 E-mail: t_kaneko@saitama-med.ac.jp

doi.org/10.2334/josnusd.58.133

DN/JST.JSTAGE/josnusd/58.133 dental treatments, intratracheal interventions, and endoscopic examination. A broad range of neuromuscular and neurological disorders, including stroke, can cause involuntary TMJ dislocation $(1,2)$. Weakness of the mouth-closing muscles innervated by the motor branch of the trigeminal nerve might be a cause of TMJ dislocation in stroke patients (2).

Stroke is the second leading cause of death worldwide, and there are many people who are suffering from poststroke complications. Stroke is a major cause of dysphagia, developing in $37-78 \%$ of cases in the early stages after stroke (3). In addition, $15 \%$ of patients still have swallowing problems, although many cases of dysphagia improve spontaneously (4). Percutaneous endoscopic gastrostomy (PEG) tube feeding is one option commonly used for patients with severe post-stroke dysphagia (5). Compared to orally fed patients, gastroesophageal reflux (GER) is known to be observed at a higher frequency among patients receiving enteral feeding after stroke, and these patients frequently develop vomiting and aspiration pneumonia (6).

This report presents a rare case of voluntary, repeated TMJ dislocation in a 73-year-old woman with post-stroke dysphagia and dysarthria. Although the dislocations initially seemed involuntary, the cause was eventually suspected to be voluntary excessive mouth opening caused by GER-related vomiting and subsequent efforts to expel oral residue of the gastric contents that had accumulated due to hypoglossal and facial nerve paralyses. Thorough oral hygiene contributed to betterthan-expected protection against TMJ dislocation. 


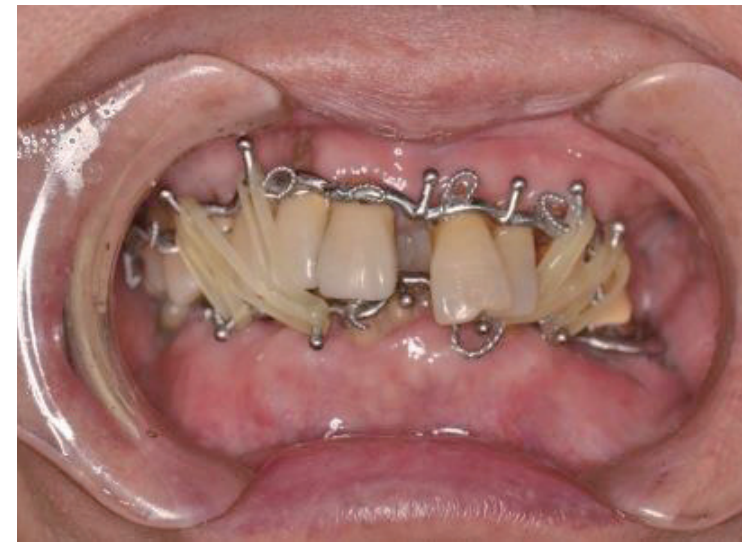

Fig. 1 Clinical view of the patient during intermaxillary fixation.

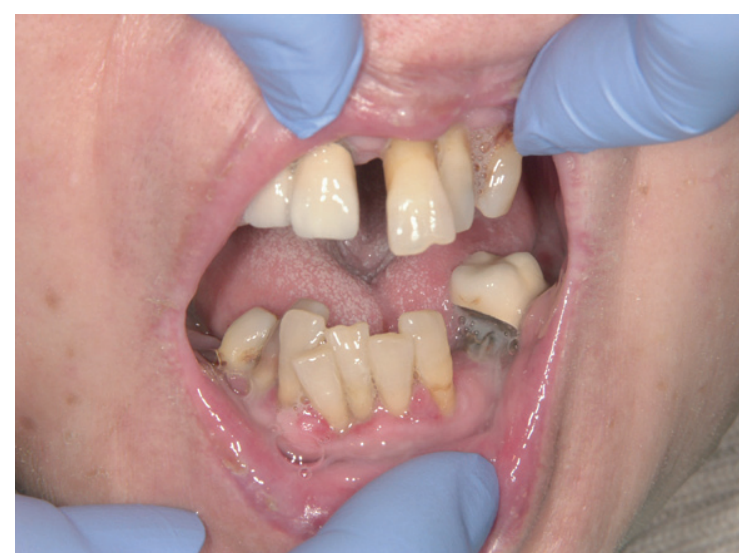

Fig. 3 Oral view after removal of the splint. No debris is found.

\section{Case Report}

A 73-year-old woman was referred to our clinic with bilateral TMJ dislocation. She was an inpatient at a nursing home, and dislocation had occurred the previous night for the first time. Caregivers in the nursing home had been unable to replace the mandible into the correct position. Two years earlier, she had suffered a left hemorrhagic hemispheric stroke that was treated conservatively. Other medical history included hypertension and diabetes mellitus (DM). Her current medications included captopril, prazosin hydrochloride, nifedipine, a Clostridium butyricum combination drug, omeprazole, and sennoside. These medications had not been changed in the preceding 6 months. Her current status showed sequelae of left hemorrhagic hemispheric stroke, including dysphagia requiring PEG, dysarthria, and right hemiparesis that required use of a wheelchair for mobility. Her degree of cognition was relatively good, but she often showed affective incontinence. On

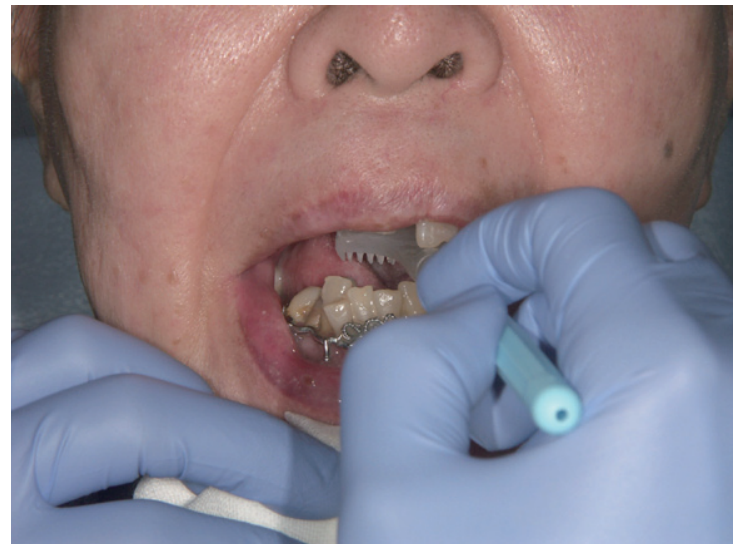

Fig. 2 Clinical view of the patient during oral hygiene activities after removal of intermaxillary rubber bands. Brushing of the tongue is being performed.

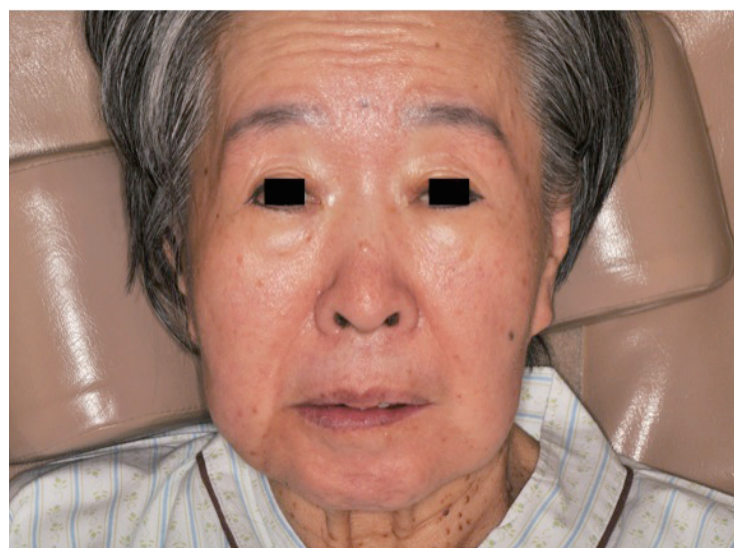

Fig. 4 Clinical view during the rehabilitation period. The patient is calmer than before and even wears makeup.

examination, bilateral TMJ dislocation was identified, and strong sour halitosis was noted. In the oral cavity, teeth $25,26,27,37,45$, and 46 were missing, and debris was evident. As she had only received PEG nutrition with no oral ingestion at all, including water, GER was suspected, with the halitosis and oral debris derived from the contents of PEG nutrition. With a diagnosis of involuntary habitual TMJ dislocation, her joints were repositioned manually. After the reposition, since the patient subsequently showed frequently repeated TMJ dislocation, it was decided to place splints on both the maxilla and mandible, and intermaxillary fixation was performed using rubber bands, because her family did not consent to operative treatment (Fig. 1). However, the rubber bands were entirely torn off, and dislocation was not stopped despite increasing the number of rubber bands used with each new dislocation. It seemed then that the patient with dysarthria was deliberately opening her mouth as a way of complaining about a bothersome 
issue. The patient's history was elicited again from her family, revealing that she had recently deliberately induced vomiting on occasion, and oral hygiene activities were less frequently performed after the first dislocation because the caregivers were afraid of causing recurrent dislocation. She had never undergone an examination of oral function. Neurological examination revealed that the patient was hardly able to move or protrude the tongue, and the tongue body was slightly deviated to the left side at rest, indicating disturbance of the right hypoglossal nerve. Likewise, she could not fully close the mouth on the right side, indicating disturbance of the right facial nerve. The sensory branches of the trigeminal nerve were undisturbed. The swallowing reflex and the emetic reflex were identified. Videoendoscopic examination of swallowing was not performed, because the family refused consent. It was speculated that the TMJ dislocation was caused by excessive mouth opening associated with GER-related vomiting and a subsequent desire to eject oral deposits accumulating due to paralyses of the hypoglossal and facial nerves. Poor oral hygiene would have contributed to inflammation on mouth opening. The dental hygienists started performing precise oral hygiene activities once a week in our clinic, and intermaxillary fixation with a decreased number of rubber bands was continued to decrease TMJ dislocation (Fig. 2). The caregivers were called so that oral hygiene might be reinforced every day. While reconsideration of the method for PEG was also requested, the procedure was not altered radically. After beginning thorough oral hygiene activities, the frequency of TMJ dislocation decreased and finally stopped, resulting in the removal of the intermaxillary fixation (Fig. 3). The patient then started rehabilitation activities for dysphagia and has shown no TMJ dislocation during the subsequent 6-month rehabilitation period (Fig. 4).

\section{Discussion}

GER during PEG feeding is associated with esophageal motor function, volume or rate of gastric infusion, gastric emptying, posture during feeding, concomitant pathologies such as DM or scleroderma, and use of drugs with the potential to alter esophageal motor function (5). In terms of esophageal motor function, relaxation of the lower esophageal sphincter (LES) has been described as a common cause of vomiting occurring early after tube feeding and associated with aspiration pneumonia. Since TMJ dislocation was found relatively early after PEG feeding, the main cause of GER was considered to be relaxation of the LES, and DM may have affected GER (7).
When GER occurred, the patient would open her mouth more than needed because of strong vomiting and the desire to remove the subsequently accumulating oral deposits, mainly attributed to the paralyses of the hypoglossal and facial nerves. In the present case, since the sensory branches of the trigeminal nerve remained undisturbed, the patient might have felt substantial discomfort with the accumulation of oral debris.

Various measures have been attempted to prevent GER associated with PEG, such as change of body position, diet feeding rate, type of nutrition, and performance of gastrojejunostomy inserted through the PEG (8). Although oral hygiene is not considered to have any direct effect on the improvement of GER, removal of residual gastric contents from GER strongly contributes to the protection of oral tissues and the prevention of aspiration pneumonia (9). In the present case, since the method of PEG could not be altered, oral hygiene was suggested as the most effective available option for protecting against TMJ dislocation.

TMJ dislocation in a post-stroke patient, though its frequency is not high, requires consideration of voluntary and involuntary causes. Patients with dysarthria may act against various stimuli in a manner sufficient to cause TMJ dislocation. In addition, implementation of oral hygiene activities in the present case with dysphagia contributed to protecting against TMJ dislocation by removing the oral debris causing GER.

\section{References}

1. Wright AJ (1985) An unusual but easily treatable cause of dysphagia and dysarthria complicating stroke. Br Med J (Clin Res Ed) 291, 1412-1413.

2. Helkimo E, Carlsson GE, Carmeli Y (1975) Bite force in patients with functional disturbances of the masticatory system. J Oral Rehabil 2, 397-406.

3. Martino R, Foley N, Bhogal S, Diamant N, Speechley M, Teasell R (2005) Dysphagia after stroke: incidence, diagnosis, and pulmonary complications. Stroke 36, 2756-2763.

4. Smithard DG, Renwick DS, Martin DF, O’Neill PA (1993) Chest infection following acute stroke: does aspiration matter? Age Ageing 22, Suppl 3, doi:10.1093/ageing/22. suppl_3.P24-a.

5. Adachi K, Furuta K, Morita T, Nakata S, Ohara S, Tanimura T et al. (2009) Half-solidification of nutrient does not decrease gastro-esophageal reflux events in patients fed via percutaneous endoscopic gastrostomy. Clin Nutr 28, 648-651.

6. Satou Y, Oguro H, Murakami Y, Onoda K, Mitaki S, Hamada $\mathrm{C}$ et al. (2013) Gastroesophageal reflux during enteral feeding in stroke patients: a 24-hour esophageal $\mathrm{pH}$-monitoring study. J Stroke Cerebrovasc Dis 22, 185-189.

7. Kuo P, Bravi I, Marreddy U, Aziz Q, Sifrim D (2013) Post- 
prandial cardiac vagal tone and transient lower esophageal sphincter relaxation (TLESR). Neurogastroenterol Motil 25, 841-e639.

8. Lawiński M, Gradowski L, Bzikowska A, Goszczyńska A, Jachnis A, Forysiński K (2014) Gastrojejunostomy inserted through PEG (PEG-J) in prevention of aspiration pneumonia. Clinical nutrition complication in dysphagic patients. Pol Przegl Chir 86, 223-229.

9. Liberali S (2008) Oral impact of gastro-oesophageal reflux disease: a case report. Aust Dent J 53, 176-179. 